Internist 2021 - 62:768-771

https://doi.org/10.1007/s00108-021-00955-9

Angenommen: 20. Januar 2021

Online publiziert: 12. Februar 2021

๑ Der/die Autor(en) 2021

\section{Redaktion}

H. Haller, Hannover (Schriftleitung)

B. Salzberger, Regensburg

C.C. Sieber, Nürnberg

\section{Anamnese}

Ein 35-jähriger Patient mit seit zwei Jahren bestehender, dialysepflichtiger Niereninsuffizienz nach orthotoper Herztransplantation bei postmyokarditischer Kardiomyopathie wurde uns aufgrund einer therapierefraktären Anämie zugewiesen.

Seit 6 Monaten bestand eine erythropoetinresistente, hyporegenerative Anämie, die mit regelmäßigen Transfusionen behandelt wurde. Im Vorfeld der stationären Aufnahme war eine Blutungsquelle mittels Gastro- und Koloskopie sowie Kapselendoskopie ausgeschlossen worden. Es bestand kein Eisenmangel, ebenso kein Mangel an Vitamin B12 oder Folsäure. Auch Antikörper gegen Erythropoetin konnten nicht nachgewiesen werden. Eine vorangegangene Knochenmarkbiopsie hatte keinen Hinweis auf ein myelodysplastisches Syndrom oder eine monoklonale Plasmazellvermehrung ergeben, zeigte jedoch eine auf $2 \%$ der Norm reduzierte Erythropoese. Eine zytologische Beurteilung entfiel bei Punctio sicca.

Seit Krankheitsbeginn hatte der $\mathrm{Pa}$ tient insgesamt $8 \mathrm{~kg}$ Körpergewicht verloren, sodass auch eine maligne Grunderkrankung denkbar war. Fieber oder eine Nachtschweißsymptomatik bestand jedoch zu keinem Zeitpunkt. Aufgrund des stark reduzierten Allgemeinzustands war der Patient nur noch am Rollator

Christoph Schröder $\cdot$ Johannes Roeles ${ }^{1}$ Adrian Schwarzer ${ }^{2}$. Michael Heuser ${ }^{2} \cdot$ Jennifer Retzlaff' $\cdot$ Marcus Hiß'

${ }^{1}$ Klinik für Nieren- und Hochdruckerkrankungen, Medizinische Hochschule Hannover, Hannover, Deutschland

${ }^{2}$ Klinik für Hämatologie, Hämostaseologie, Onkologie und Stammzelltransplantation, Medizinische Hochschule Hannover, Hannover, Deutschland

\title{
Therapierefraktäre Anämie bei einem 35-jährigen Dialysepatienten nach Herztransplantation
}

mobil. Er ist Vater von drei gesunden Kindern im schulpflichtigen Alter. Das immunsuppressive Regime bestand aus Everolimus, Tacrolimus und Prednisolon p.o.

\section{Klinischer Befund}

Der Patient präsentierte sich dyspnoisch mit blassem Hautkolorit. Das Körpergewicht lag bei $80 \mathrm{~kg}$ bei einer Körpergröße von $185 \mathrm{~cm}$ (Body-Mass-Index $23,7 \mathrm{~kg} / \mathrm{m}^{2}$ ). Der Blutdruck war mit 108/61 mm Hg erniedrigt, die Herzfrequenz mit 103/min erhöht. Es bestand kein Fieber $\left(37,1^{\circ} \mathrm{C}\right)$, die pulsoxymetrisch gemessene Sauerstoffsättigung betrug $100 \%$. Das Integument zeigte sich unauffällig, es bestand eine Euvolämie.

\section{Diagnostik}

Im Aufnahmelabor zeigte sich eine normochrome, hyporegenerative Anämie (Hämoglobin 7,6g/dl) mit einer stark erniedrigten Retikulozytenzahl (2/nl; Norm 25-105/nl; Retikulozytenproduktionsindex 0). Thrombozyten und Leukozyten waren normwertig, im Differenzialblutbild zeigte sich eine isolierte Lymphozytopenie. Darüber hinaus bestand eine Eisenüberladung (Ferritin $800 \mathrm{ng} / \mathrm{ml}$, Transferrinsättigung $50 \%$ ), ein Vitamin-B12- oder Folsäuremangel konnte ausgeschlossen werden. Das C-reaktive Protein war mit 7,3 mg/l nur leicht erhöht. Serologisch fand sich ein positiver Parvovirus-B19-ImmunglobulinM(IgM)-Titer, bei negativem Parvovirus-B19-Immunglobulin G (IgG). Aktive Infektionen durch das Zytomegalievirus und Epstein-Barr-Virus wurden ausgeschlossen. Eine Abdomensonographie erbrachte einen Normalbefund.

\section{Differenzialdiagnosen}

- Differenzialdiagnostisch wurden zu diesem Zeitpunkt eine medikamentös-toxische Knochenmarkschädigung (insbesondere mTORInhibitor-vermittelt) und eine infektiologische Ursache (vor allem eine Virusinfektion) in Betracht gezogen.

\section{Weiterer Verlauf}

Die Everolimusbehandlung wurde pausiert, ein Hydrokortisonperfusor etabliert. Die Knochenmarkaspiration wurde wiederholt. In der zytologischen Untersuchung des Knochenmarks zeigte sich ein hypozelluläres Mark mit einer hochgradigen Reduktion der ausreifenden Erythropoese bei normaler Granulound Megakaryopoese. Auffällig war der Nachweis von Riesenproerythroblasten (Gigantoblasten) mit nukleären Viruseinschlüssen (• Abb. 1). Zusätzlich konnte mittels Polymerase-Kettenreaktion (PCR) eine Parvoviruslast von $80 \mathrm{Mrd}$. $\mathrm{IU} / \mathrm{ml} \mathrm{im}$ Serum und $50 \mathrm{Mrd}$. IU/ml im 


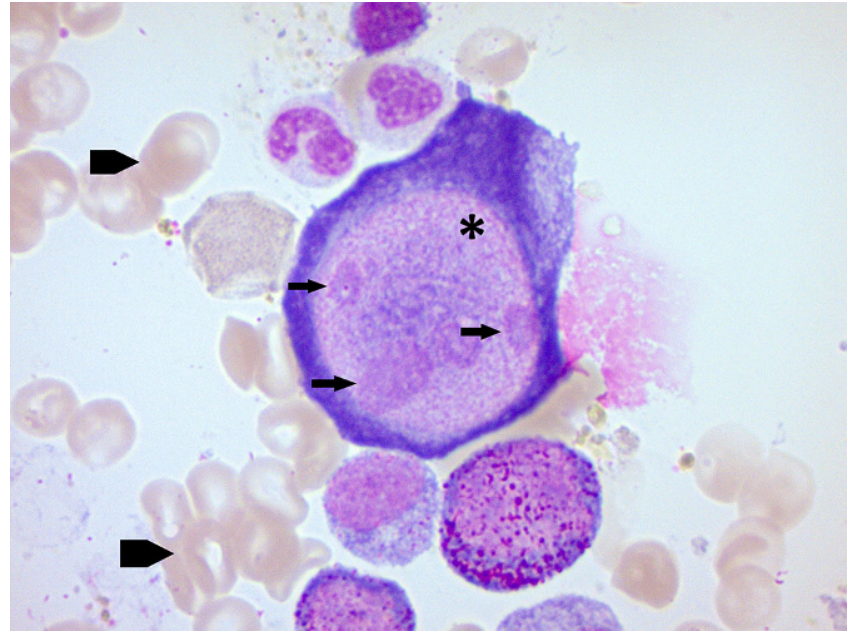

Abb. $1 \triangleleft$ Knochenmarkausstrich des vorgestellten Patienten. Riesenproerythroblast (Asterisk) mit eosinophilen nukleären Einschlüssen (Pfeile). Im Größenvergleich dazu reife Erythrozyten (Pfeilköpfe). Ein vollständiger Stopp der Erythropoese auf dem $\mathrm{Ni}$ veau des Proerythroblasten ist erkennbar

Knochenmark gesichert werden. Im weiteren stationären Verlauf aggravierte die Anämiesymptomatik unter einem Hämoglobinwert von 6,4 g/dl.

\section{Diagnose}

- „Pure red cell aplasia“ (PRCA) bei Parvovirus-B19-Infektion

\section{Therapie und Verlauf}

Eine hoch dosierte Immunglobulintherapie mit $2 \mathrm{~g} / \mathrm{kg}$ über 5 Tage wurde initiiert. Zum Zeitpunkt der Entlassung erfolgte die Reduktion der Everolimus- und Tacrolimusdosis unter vorübergehender Erhöhung des oralen Prednisolons.

Vier Wochen später stellte sich der Patient in gutem Allgemeinzustand vor.
Der Hämoglobinwert zeigte sich mit $10,8 \mathrm{~g} / \mathrm{dl}$ gebessert, ohne zwischenzeitlichen Transfusionsbedarf. Die Parvovirus-B19-Viruslast im Serum war mit $3000 \mathrm{IU} / \mathrm{ml}$ rückläufig, eine ParvovirusB19-IgM-zu-IgG-Serokonversion hatte stattgefunden.

\section{Diskussion}

Krankheitsbild der Parvovirus-B19Infektion

Eine Infektion mit dem Parvovirus B19 verläuft bei immunkompetenten Patienten in der Regel asymptomatisch. Die häufigste klinische Manifestation ist im Kindesalter das Erythema infectiosum. Auf ein unspezifisches Prodromalstadium mit Fieber, Schüttelfrost, Zephalgien und Myalgien folgen ein schmetterlingsförmiges Gesichtserythem („slapped cheek") und ein girlandenförmiges Ganzkörperexanthem [10].

Hier steht eine Anzeige. 
I) Nach Organtransplantation ist das Risiko der Chronifizierung einer Parvovirus-B19-Infektion erhöht

Bei Patienten mit chronischen Vorerkrankungen zeigt sich jedoch oft ein hiervon abweichender klinischer Verlauf [9]. Insbesondere Patienten nach Organtransplantation haben ein erhöhtes Risiko für eine Chronifizierung der Infektion. Die weitaus häufigste Manifestation innerhalb dieses Patientenkollektivs ist eine progrediente Anämie mit körperlicher Schwäche, Dyspnoe und orthostatischer Dysregulation [2, 5]. Ein Ganzkörperexanthem tritt in nur 13\% der Fälle auf. Etwas seltener zeigen sich auch andere Organmanifestationen wie eine Myokarditis, eine Pneumonie oder Hepatitis. Zu einer Transplantatdysfunktion oder zum Transplantatverlust kommt es in etwa $10 \%$ der Fälle. Hier ist die Dysfunktion des Nierentransplantats mit $15,6 \%$ führend, gefolgt von Herzund Lungentransplantaten mit 8,3\% [5].

Die Virusübertragung erfolgt mittels Tröpfcheninfektion. Auf eine Virusreplikation im Nasopharyngealraum folgt etwa eine Woche später eine Virämie [1]. Die Virusreplikation in erythropoetischen Progenitorzellen führt dann zur Herunterregulation von Erythropoetinrezeptoren, zum Zellzyklusarrest und letztlich zur Apoptose der Zelle [6].

\section{Differenzialdiagnose bei anämischen Patienten nach Organtransplantation}

Generell sollten bei Patienten nach Organtransplantation, die eine Anämie aufweisen, neben unspezifischen Ursachen wie einem Mangel an Eisen, Vitamin B12 oder Folsäure auch transplantationsspezifische Ursachen ausgeschlossen werden. Hierzu gehören unter anderem medikamentös-toxische Knochenmarkschädigungen. Diese können zum einen durch immunsuppressive Substanzen wie Azathioprin, Mycophenolatmofetil oder mTOR-Inhibitoren bedingt sein, aber auch durch antimikrobielle Prophy-

Internist 2021 -62:768-771 https://doi.org/10.1007/s00108-021-00955-9

(c) Der/die Autor(en) 2021

C. Schröder · J. Roeles · A. Schwarzer · M. Heuser · J. Retzlaff · M. Hiß

Therapierefraktäre Anämie bei einem 35 -jährigen Dialysepatienten nach Herztransplantation

\section{Zusammenfassung}

Es wird über einen 35-jährigen Dialysepatienten nach Herztransplantation berichtet, der eine "pure red cell aplasia“ aufwies. Serologisch fand sich ein positiver Parvovirus-B19Immunglobulin-M-Titer, im Direktnachweis mittels Polymerase-Kettenreaktion zeigten sich $80 \mathrm{Mrd}$. IU/ml. In der zytologischen Untersuchung des Knochenmarks gelang der Nachweis von Riesenproerythroblasten, pathognomonisch für eine Parvovirus-B19Infektion. Zur Therapie erfolgte die hoch dosierte Gabe von Immunglobulinen über
5 Tage. Bei Wiedervorstellung nach vier Wochen zeigte sich die Anämie deutlich gebessert. Bei Patienten nach Organtransplantation mit hyporegenerativer Anämie sollte eine Parvovirus-B19-Infektion stets ausgeschlossen werden.

Schlüsselwörter

Parvovirus B19 · Isolierte aplastische Anämie . Riesenproerythroblasten · Intravenöse Immunglobuline P Polymerase-Kettenreaktion

\section{Treatment-refractory anaemia in a 35-year-old heart transplant recipient on chronic hemodialysis}

\section{Abstract}

This article presents a case of pure red cell aplasia in a 35-year-old heart transplant recipient on chronic hemodialysis. Elevated parvovirus B19 immunoglobulin M blood levels were detected along with a high viral load of 80 billion IU/ml quantified by polymerase chain reaction. Bone marrow examination revealed giant proerythroblasts confirming parvovirus B19 infection. Highdose intravenous immunoglobulin was used for treatment. Anaemia had significantly improved 4 weeks later. Parvovirus B19 infection should be excluded in organ transplant recipients with anaemia due to ineffective erythropoiesis.

Keywords

Parvovirus B19, human · Red cell aplasia, pure - Megaloblasts - Immunoglobulins, intravenous · Polymerase chain reaction laxen, beispielsweise mit Trimethoprim [8].

Des Weiteren sind chronische Infektionen in Erwägung zu ziehen. Insbesondere virale Erreger spielen eine wichtige Rolle. Neben dem Parvovirus B19 ist an eine Infektion mit dem Zytomegalievirus oder dem Epstein-Barr-Virus zu denken, seltener auch an das Varizella-Zoster-Virus, das humane Herpesvirus 6 und 8 sowie an das BK-Virus. Auch nichtinfektiöse Erkrankungen wie das Posttransplantationslymphom oder eine thrombotische Mikroangiopathie sollten differenzialdiagnostisch in Erwägung gezogen werden [8].

\section{Diagnostik der Parvovirus-B19- Infektion}

Zum Ausschluss einer Parvovirus-B19Infektion sollte die serologische Initialdiagnostik um eine PCR ergänzt werden [4], da die antikörpervermittelte Immunantwort inadäquat oder verzögert verlaufen kann. In einem Kollektiv von 98 Patienten mit Parvovirus-B19-Infektion nach Transplantation zeigten $29 \%$ initial eine negative IgM-Serologie [5]. Der Virusdirektnachweis aus infiziertem Gewebe, beispielsweise der Niere, Leber, Lunge oder des Knochenmarks, kann bei negativem serologischem Nachweis und fortbestehendem Verdacht sinnvoll sein. In der zytologischen Untersuchung des 
Knochenmarks zeigt sich eine hochgradige Reduktion bis hin zum völligen Fehlen von Vorstufen der Erythropoese. Häufig lassen sich charakteristische Riesenproerythroblasten mit eosinophilen nukleären Einschlüssen nachweisen [7].

\section{Therapie}

Eine spezifische antivirale Therapie existiert derzeit nicht. Therapeutisch kommt die intravenöse Gabe von Immunglobulinen (IVIG) in hoher Dosierung zum Einsatz. Die American Society of Transplantation empfiehlt eine Dosierung von $400 \mathrm{mg} / \mathrm{kg}$ täglich. Falls möglich sollte eine Reduktion der Immunsuppression erfolgen [4]. Als Nebenwirkungen können Fieber, Schüttelfrost, Zephalgien, Myalgien, Übelkeit, Blutdruckentgleisungen, Angina pectoris und eine akute Nierenfunktionsverschlechterung auftreten. Die Verabreichung der Gesamtdosis über 5 Tage im Vergleich zu einem 2-tägigen Therapieregime erhöht die IVIG-Verträglichkeit [3].

\section{》) Therapeutisch kommt die intravenöse Gabe von Immunglobulinen in hoher Dosierung zum Einsatz}

Die Ansprechrate der PRCA auf die IVIG-Behandlung liegt bei $93 \%$ [3]. Jedoch gibt es zurzeit keine prospektiven, kontrollierten Untersuchungen, die den Effekt dieser Behandlung beweisen. In einzelnen kleinen Kohorten von Patienten mit PRCA bei Parvovirus-B19Infektion nach Transplantation beträgt die spontane Remissionsrate etwa $60 \%$ $[2,5]$. Die Rezidivrate der ParvovirusB19-assoziierten PRCA liegt bei 28-34\%, unabhängig von der Immunglobulingesamtdosis ( $\leq 2 \mathrm{~g} / \mathrm{kg}$ oder $>2 \mathrm{~g} / \mathrm{kg} ;[3,5])$. Die American Society of Transplantation empfiehlt in diesen Fällen weitere IVIGZyklen in 4-wöchigen Abständen bis zum Sistieren der Symptomatik [4].

Der Stellenwert der PCR in der Verlaufskontrolle ist nicht gesichert. In seltenen Fällen kann die Virus-DNA bis zu 3 Jahre nach dem akuten Infekt nachgewiesen werden. Eine solche „DNAämie“ sollte lediglich zur Veranlassung einer regelmäßigen Hämoglobinmessung führen, beim Auftreten einer erneuten Anämie sollte eine IVIG-Behandlung erfolgen [4].

\section{Fazit für die Praxis}

- Eine Parvovirusinfektion manifestiert
sich bei Patienten nach Organtrans-
plantation am häufigsten als Anämie.
- Die initiale serologische Diagnostik
sollte in diesem Patientenkollektiv
um eine Polymerase-Kettenreaktion
(PCR) ergänzt werden.
Therapeutisch kommen Immun-
globuline in einer Dosierung von
400 mg/kg täglich über 5 Tage zum
Einsatz, falls möglich sollte eine
Reduktion der Immunsuppression
erfolgen.
Das Therapiemonitoring sollte in
Bezug auf die klinische Symptomatik
erfolgen, der Stellenwert der PCR
in der Verlaufskontrolle ist nicht
gesichert.

\section{Korrespondenzadresse}

\section{Dr. Christoph Schröder}

Klinik für Nieren- und Hochdruckerkrankungen, Medizinische Hochschule Hannover Carl-Neuberg-Straße 1, 30625 Hannover, Deutschland

schroeder.christoph.nph@mh-hannover.de

Funding. Open Access funding enabled and organized by Projekt DEAL.

\section{Einhaltung ethischer Richtlinien}

Interessenkonflikt. C. Schröder, J. Roeles, A. Schwarzer, M. Heuser, J. Retzlaff und M. Hiß geben an, dass kein Interessenkonflikt besteht.

Für diesen Beitrag wurden von den Autoren keine Studien an Menschen oder Tieren durchgeführt. Für die aufgeführten Studien gelten die jeweils dort angegebenen ethischen Richtlinien. Für Bildmaterial oder anderweitige Angaben innerhalb des Manuskripts, über die Patienten zu identifizieren sind, liegt von ihnen und/oder ihren gesetzlichen Vertretern eine schriftliche Einwilligung vor.

Open Access. Dieser Artikel wird unter der Creative Commons Namensnennung 4.0 International Lizenz veröffentlicht, welche die Nutzung, Vervielfältigung, Bearbeitung, Verbreitung und Wiedergabe in jeglichem Medium und Format erlaubt, sofern Sie den/die ursprünglichen Autor(en) und die Quelle ordnungsgemäß nennen, einen Link zur Creative Commons Lizenz beifügen und angeben, ob Änderungen vorgenommen wurden.

Die in diesem Artikel enthaltenen Bilder und sonstiges Drittmaterial unterliegen ebenfalls der genannten Creative Commons Lizenz, sofern sich aus der Abbildungslegende nichts anderes ergibt. Sofern das betreffende Material nicht unter der genannten Creative Commons Lizenz steht und die betreffende Handlung nicht nach gesetzlichen Vorschriften erlaubt ist, ist für die oben aufgeführten Weiterverwendungen des Materials die Einwilligung des jeweiligen Rechteinhabers einzuholen.

Weitere Details zur Lizenz entnehmen Sie bitte der Lizenzinformation auf http://creativecommons.org/ licenses/by/4.0/deed.de.

\section{Literatur}

1. Anderson MJ, Higgins PG, Davis LR, Willman JS, Jones SE, Kidd IM, Pattison JR, Tyrrell DA (1985) Experimental parvoviral infection in humans. JInfect Dis 152(2):257-265

2. Broliden K (2001) Parvovirus B19 infection in pediatric solid-organ and bone marrow transplantation. Pediatr Transplant 5(5):320-330

3. Crabol Y, Terrier B, Rozenberg F, Pestre V, Legendre C, Hermine O, Montagnier-Petrissans $C$, Guillevin L, Mouthon L (2013) Intravenous immunoglobulin therapy for pure red cell aplasia related to human parvovirus b19 infection: a retrospective study of 10 patients and review of the literature. Clin Infect Dis 56(7):968-977

4. Eid AJ, Ardura MI (2019) Human parvovirus B19 in solid organ transplantation: guidelines from the American society of transplantation infectious diseases community of practice. Clin Transplant 33(9):e13535

5. Eid AJ, Brown RA, Patel R, Razonable RR (2006) Parvovirus B19 infection after transplantation: a review of 98 cases. Clin Infect Dis 43(1):40-48

6. Guo Y-M, Ishii K, Hirokawa M, Tagawa H, Ohyagi H, Michishita Y, Ubukawa K, Yamashita J, Ohteki T, Onai N, Kawakami K, Xiao W, Sawada K (2010) CpGODN 2006 and human parvovirus B19 genome consensus sequences selectively inhibit growth and development of erythroid progenitor cells. Blood 115(22):4569-4579

7. Mende M, Sockel K (2018) Parvovirus B19 Infection. NEngl JMed 379(24):2361

8. Reindl-Schwaighofer R, Oberbauer R (2014) Blood disorders after kidney transplantation. Transplant Rev 28(2):63-75

9. Sim JY, Chang L-Y, Chen J-M, Lee P-I, Huang L-M, Lu C-Y (2019) Human parvovirus B19 infection in patients with or without underlying diseases. JMicrobiol Immunol Infect 52(4):534-541

10. Young NS, Brown KE (2004) Parvovirus B19. N Engl J Med 350(6):586-597 